\title{
An Epidemiological Cohort Study of SARS-CoV-2 and COVID-19 in German Healthcare Workers - Interim Analysis after Six Months of Follow-up
}

\section{Stephan Gehring ( $\sim$ Stephan.Gehring@uni-mainz.de)}

Universitätsmedizin Mainz Zentrum für Kinder- und Jugendmedizin

\section{Omar Okasha}

P95, Pharmacovigilance and Epidemiology Koning Leopold III laan 13001 Leuven Belgium

Frank Kowalzik

Universitätsmedizin Mainz Zentrum für Kinder- und Jugendmedizin

\section{Tobias Engelmann}

Universitätsmedizin Mainz Zentrum für Kinder- und Jugendmedizin

\section{Daniel Schreiner}

Universitätsmedizin Mainz Zentrum für Kinder- und Jugendmedizin

Christian Jensen

Universitätsmedizin Mainz Zentrum für Kinder- und Jugendmedizin

Aline Maehringer-Kunz

Universitätsmedizin Mainz Zentrum für Kinder- und Jugendmedizin

\section{Wendy Hartig-Merkel}

P95, Pharmacovigilance and Epidemiology Koning Leopold III laan 13001 Leuven Belgium

Anirudh Tomer

P95, Pharmacovigilance and Epidemiology Koning Leopold III laan 13001 Leuven Belgium

\section{Lidia Oostvogels}

CureVac (Germany)

Thomas Verstraeten

P95, Pharmacovigilance and Epidemiology Koning Leopold III laan 13001 Leuven Belgium

\section{Research Article}

Keywords: Healthcare workers (HCWs), SARS-CoV-2 infection, COVID-19 vaccines

Posted Date: August 3rd, 2021

DOI: https://doi.org/10.21203/rs.3.rs-688656/v1 
License: (c) (i) This work is licensed under a Creative Commons Attribution 4.0 International License. Read Full License 


\section{Abstract}

\section{Background}

Healthcare workers (HCWs) are at increased risk of SARS-CoV-2 infection. We assessed incidence of SARS-CoV-2 infection and COVID-19 before the roll out of COVID-19 vaccines in a cohort of HCWs in Mainz, Germany.

\section{Methods}

Using prospective observational cohort design, antibody status was assessed at baseline and every 6 weeks ( \pm 2 weeks). Daily self-reported COVID-19 symptoms were collected using a smartphone application. Symptomatic HCWs were tested using RT-PCR. We estimated symptomatic and asymptomatic SARS-CoV-2 infection rates based on two case definitions of varying sensitivity and specificity.

\section{Results}

3664 HCWs were enrolled with a median follow-up of 101 days. The seroprevalence of anti-SARS-CoV-2 antibodies increased from $2.7 \%$ at baseline to $3.8 \%$, with the number of seroconversions (65) outweighing seroreversions (26) by end of follow-up. Among HCWs who seroconverted, 12 ( 19\%) did not report any symptoms. The estimated incidence rate was 4.5 per 1000 person-months, but none the incident cases developed severe disease. Anti-SARS-CoV-2 antibodies fell below diagnostic cut-off value in a third of those positive at baseline and in one incident case.

\section{Conclusions}

We observed increasing COVID-19 rates among HCWs during an accelerated community transmission period, with relatively lower rate of asymptomatic infections. Our findings indicate a relatively long-lasting humoral immune response following natural infection.

\section{Introduction}

In December 2019, the novel SARS-CoV-2 coronavirus was isolated from a cluster of patients presenting with pneumonia with epidemiological links to a seafood and wet animal market in Wuhan, Hubei Province, China. ${ }^{1}$ By the end of January 2020 , there were $~ 10,000$ confirmed cases in 20 countries with all deaths (213) reported in China. The WHO designated the disease caused by SARS-CoV-2 as COVID-19, and on 12 March 2020, it was declared a pandemic. ${ }^{2}$

As of 26 May 2021, > 167 million cases were confirmed globally, and the number of deaths exceeded 3.4 million. ${ }^{3}$ Nearly a third of all confirmed cases and deaths were reported in Europe where $>53$ million confirmed cases and >1.1 million deaths were reported. In Germany, the majority of the $~ 3.6$ million 
cases and 87,700 deaths were reported during the second wave of the epidemic, which entered the exponential growth phase in August-September 2020. ${ }^{3}$

Healthcare workers (HCWs) are at increased risk of SARS-CoV-2 infection given their exposure via hospitalized COVID-19 patients, subclinical infections, and community transmission. ${ }^{4}$ Results from a recent meta-analysis indicate that up to $40 \%$ (95\% Cl: 17-65\%) of SARS-CoV-2 infected HCWs were asymptomatic at the time of screening. ${ }^{5}$ Although HCWs are considered a priority group for which a number of COVID-19 vaccines have been recommended under emergency or conditional marketing authorization, ${ }^{6}$ vaccine hesitancy remains a significant challenge to ongoing immunization programs. ${ }^{7}$

Few studies to date assessed the risk and clinical characteristics of SARS-CoV-2 infection in HCWs using prospective designs. We report results from the 'CPMprevac' cohort study, which aims to assess the rates of both symptomatic and asymptomatic SARS-CoV-2 infection in a cohort of HCWs at a tertiary care center in Germany.

\section{Results}

\section{Population and follow-up}

A total of $3664 \mathrm{HCWs}$ were enrolled in the study between August - October 2020. The study subjects contributed approximately 12,000 person months of follow-up, with a median follow-up duration of 101 days (Interquartile range (IQR) 89-110 days). The mean age of study subjects at enrolment was 39 years; $>70 \%$ were younger than 50 years (Table 1) and two thirds were females. Nurses, doctors, and medical students constituted the majority of study subjects $(29.6 \%, 14.4 \%$, and $16.7 \%$, respectively). Approximately $15 \%$ of subjects were current or former smokers, $\sim 17 \%$ had chronic lung and/or heart disease, $15 \%$ reported direct care of confirmed COVID-19 cases, and 10\% reported contact with possible cases.

\section{Table 1}

Baseline characteristics of the study population in the CPMprevac study, UM Mainz, Germany (August

$$
2020 \text { - January 2021) }
$$




\begin{tabular}{|c|c|c|c|c|c|}
\hline & \multirow[t]{3}{*}{$\begin{array}{l}\text { Sub- } \\
\text { group }\end{array}$} & \multirow{3}{*}{ 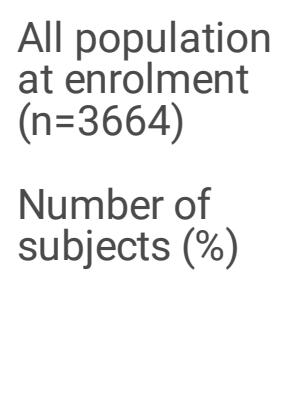 } & \multicolumn{2}{|c|}{$\begin{array}{l}\text { Population characteristics, by } \\
\text { seroconversion status }\end{array}$} & \multirow{3}{*}{$p$} \\
\hline & & & $\begin{array}{l}\text { Did not } \\
\text { seroconvert }\end{array}$ & $\begin{array}{l}\text { Seroconverted } \\
(n=65)\end{array}$ & \\
\hline & & & $\begin{array}{l}\text { Number of } \\
\text { subjects }(\%)\end{array}$ & $\begin{array}{l}\text { Number of } \\
\text { subjects (\%) }\end{array}$ & \\
\hline \multirow[t]{6}{*}{ Age (years) } & $\begin{array}{l}\text { Mean } \\
\text { (SD) }\end{array}$ & $39.1(13.4)$ & $39.2(13.4)$ & $33.3(12.5)$ & NA \\
\hline & $<29$ & $1114(30.4)$ & $1078(30.0)$ & $34(52.3)$ & \multirow[t]{5}{*}{0.004} \\
\hline & $30-39$ & $897(24.5)$ & $885(24.6)$ & $11(16.9)$ & \\
\hline & $40-49$ & $599(16.3)$ & $590(16.4)$ & $8(12.3)$ & \\
\hline & $50-59$ & $719(19.6)$ & $710(19.8)$ & $8(12.3)$ & \\
\hline & $>60$ & $335(9.1)$ & $331(9.2)$ & $4(6.2)$ & \\
\hline \multirow[t]{3}{*}{ Gender } & Female & $2760(75.3)$ & $2708(75.3)$ & $49(75.4)$ & \multirow[t]{3}{*}{0.973} \\
\hline & Male & $902(24.6)$ & $883(24.6)$ & $16(24.6)$ & \\
\hline & Other & $3(0.1)$ & $3(0.1)$ & $0(0.0)$ & \\
\hline \multirow[t]{4}{*}{ Occupational group } & Doctor & $529(14.4)$ & $522(14.5)$ & $7(10.8)$ & \multirow[t]{4}{*}{0.344} \\
\hline & Nursing & $1084(29.6)$ & $1063(29.6)$ & $18(27.7)$ & \\
\hline & Student & $612(16.7)$ & $593(16.5)$ & $16(24.6)$ & \\
\hline & Other & $1440(39.3)$ & $1416(39.4)$ & $24(36.9)$ & \\
\hline Current or past smoker & & $555(15.1)$ & $546(15.2)$ & $7(10.8)$ & 0.416 \\
\hline $\begin{array}{l}\text { Disorders of airways and/or } \\
\text { lung }\end{array}$ & & $345(9.4)$ & $339(9.5)$ & $5(7.7)$ & 0.790 \\
\hline $\begin{array}{l}\text { Disorders of the } \\
\text { cardiovascular system }\end{array}$ & & $300(8.2)$ & $297(8.3)$ & $3(4.6)$ & 0.403 \\
\hline $\begin{array}{l}\text { Immunosuppressing or } \\
\text { immunocompromising } \\
\text { conditions }\end{array}$ & & $96(2.6)$ & $94(2.6)$ & $2(3.1)$ & 1.000 \\
\hline $\begin{array}{l}\text { Direct care for confirmed } \\
\text { SARS-CoV- } 2 \text { patients }\end{array}$ & & $532(14.5)$ & $521(14.5)$ & $10(15.4)$ & 0.984 \\
\hline $\begin{array}{l}\text { Direct contact with a } \\
\text { possible SARS-CoV-2 } \\
\text { patient }\end{array}$ & & $369(10.1)$ & $363(10.1)$ & $6(9.2)$ & 0.982 \\
\hline $\begin{array}{l}\text { Travelled to a SARS-CoV-2 } \\
\text { area }\end{array}$ & & 3567 (97.3) & 3498 (97.4) & 63 (96.9) & 1.000 \\
\hline
\end{tabular}




\section{SARS-CoV-2 infection}

\section{Seroprevalence}

Of 3664 HCWs with available baseline serological data on anti-SARS-CoV-2 antibodies, 98 (2.7\%; $95 \%$ Cl: 2.2\%-3.3\%) were seropositive for anti-SARS-CoV-2 IgM and/or IgG antibodies (Table 2). The

seroprevalence estimates increased to $3.3 \%$ (95\% Cl: $2.7 \%-3.9 \%$ ) and 3.8\% (95\% Cl: $3.2 \%-4.6 \%)$ at the 2nd and 3rd follow-up visits, respectively. Adjusting for diagnostic misclassification resulted in slightly lower seroprevalence estimates (Table 2).

Table 2

SARS-CoV-2 infection in healthcare workers in the CPMprevac study, UM Mainz, Germany (August 2020 - January 2021) 
Baseline $\quad$ Follow-up visit $2 \quad$ Follow-up visit 3

Seroprevalence

\begin{tabular}{|c|c|c|c|}
\hline Number of subjects with serology data & 3664 & 3605 & 3012 \\
\hline Number of seropositive subjects* & $98^{\star \star \star}$ & 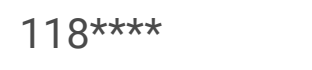 & 115 \\
\hline Crude seroprevalence & $2.67 \%$ & $3.27 \%$ & $3.82 \%$ \\
\hline$[95 \% \mathrm{Cl}]$ & {$[2.18 \%, 3.25 \%]$} & {$[2.72 \%, 3.91 \%]$} & {$[3.16 \% ; 4.57 \%]$} \\
\hline \multirow[t]{2}{*}{ Adjusted seroprevalence ${ }^{\star \star}[95 \% \mathrm{Cl}]$} & $1.89 \%$ & $2.49 \%$ & $3.04 \%$ \\
\hline & {$[1.39 \%, 2.47 \%]$} & {$[1.93 \%, 3.13 \%]$} & {$[2.38 \% ; 3.80 \%]$} \\
\hline \multicolumn{4}{|l|}{ Seroconversion at follow-up visits } \\
\hline Number of subjects at risk ${ }^{\star \star \star \star \star}$ & NA & 3509 & 2921 \\
\hline Number of subjects who seroconverted & NA & 33 & 32 \\
\hline Crude seroconversion & NA & $0.94 \%$ & $1.1 \%$ \\
\hline$[95 \% \mathrm{Cl}]$ & & {$[0.65 \% ; 1.32 \%]$} & {$[0.75 \% ; 1.5 \%]$} \\
\hline \multirow[t]{2}{*}{ Adjusted seroconversion ${ }^{\star \star}[95 \% \mathrm{Cl}]$} & NA & $0.14 \%$ & $0.30 \%$ \\
\hline & & {$[-0.15 \% ; 0.52 \%]$} & {$[-0.05 \% ; 0.75 \%]$} \\
\hline
\end{tabular}

* Positive serostatus is defined as being seropositive on either or both IgG and IgM.

** Adjusted for diagnostic misclassification by accounting for the sensitivity and specificity of the tests.

*** Out of 98 seropositive healthcare workers at baseline, 12 subjects later seroreverted by follow-up visit 2 and 14 seroreverted by follow-up visit 3 .

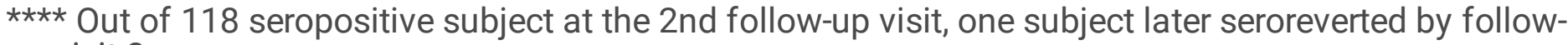
up visit 3 .

$\star \star \star \star \star$ Subjects at risk of seroconversion are defined as those with negative serostatus at the previous visit and available data on serostatus at the subsequent visit.

Abbreviations: Cl: confidence interval; NA: not applicable.

\section{Seroconversion}

Of $3509 \mathrm{HCWs}$ with both negative serostatus at baseline and available serostatus data at the 2nd visit, 33 (0.94\%) subjects seroconverted at the 2 nd visit (95\% Cl: $0.65 \%$ to $1.32 \%)$. Similarly, $32 / 2921(1.10 \%)$ seroconverted at the $3 \mathrm{rd}$ visit ( $95 \% \mathrm{Cl}: 0.75 \%$ to $1.57 \%)$. Among the 65 subjects who seroconverted, 12 
(18.5\%) did not report any symptoms during the study period. Subjects who seroconverted were relatively younger than those who did not, however, the two groups were similar with regards to other baseline characteristics (Table 1).

\section{Seroreversion}

Of the 98 subjects who were seropositive at baseline, 11 and 15 subjects seroreverted by the 2nd and 3rd follow-up visits, respectively. In addition, one subject of the 33 subjects who seroconverted at the 2 nd visit reverted by the next visit (Supplementary Figure S.4). None of the 27 subjects who seroreverted had evidence of reinfection occurring at any point after 60 days had passed from their negative serological test.

\section{COVID-19 disease}

\section{Incidence}

Based on the US FDA case definition, a total of 53 virologically confirmed COVID-19 cases were identified, which translates to a cumulative incidence of 14.5 per 1000 persons ( $95 \% \mathrm{Cl}: 10.9-18.9)$ and an incidence density of 4.5 per 1000 person-months ( $95 \% \mathrm{Cl}$ : 3.3-5.8). The mean age of incident cases was 35.4 years (SD 14.2) and $>38 \%$ were nurses. None of the incident cases fulfilled either case definition of severe COVID-19 disease. However, two cases had pneumonia, of which one required hospitalization (Table 3). Applying the more specific case definition by CEPI yielded slightly lower estimates, with a cumulative incidence proportion of 11.2 per 1000 persons (95\% Cl: 8.0-15.2) and density of 3.4 per 1000 person months (95\%. Cl: 2.5-4.6).

\section{Table 3}

Characteristics of 55 virologically confirmed COVID-19 cases among healthcare workers in the CPMprevac study, UM Mainz, Germany (August 2020 - January 2021) 
Number (\%)

Gender

Female

Male

Age (years)

mean (SD)

$\leq 29$

$30-39$

$40-49$

$50-59$

$\geq 60$

Occupational Group

Doctor

Nursing

Student

Other*

Exposures in the 6 weeks preceding interview

Travelled to SARS-CoV-2 area

Contact with confirmed SARS-CoV-2 patient

Smoking status

Does not smoke

Still smoking

Stopped smoking

Chronic Lung Disease

Chronic Heart Disease

Immunosuppressing or immunocompromising condition

Complications of COVID-19

Pneumonia

Hospitalization
$40(72.7)$

$15(27.3)$

$35.39(14.15)$

$24(43.6)$

$10(18.2)$

$8(14.5)$

$9(16.4)$

$4(7.3)$

$4(7.3)$

$21(38.2)$

$11(20.0)$

19 (34.5)

2 (3.6)

29 (52.7)

51 (92.7)

3 (5.5)

1 (1.8)

1 (1.8)

2 (3.6)

2 (3.6)

1 (1.8) 
*Other complications include intensive care unit (ICU) admission, mechanical ventilation, extracorporeal membrane oxygenation (ECMO), renal failure, liver failure, neurological dysfunction, heart failure, hypotension, and coagulopathy.

An additional 30 subjects had positive RT-PCR but did not have sufficient data to be classified as COVID19 cases. Of these, 23 reported symptoms via the mobile application - of whom 6 subjects were also found to seroconvert. Of the remaining 7 RT-PCR positive subjects who were asymptomatic, two seroconverted. Taking these additional 30 cases into account results in a cumulative incidence estimate of 22.7 per 1000 persons.

\section{Symptoms of COVID-19}

A total of 1804 episodes of COVID-19-related symptoms were reported by 1207 subjects throughout the study period, of which only 55 ( 3\%) episodes - reported by 55 subjects - were virologically confirmed. Among confirmed COVID-19-positive episodes, the two most common symptoms reported were cough and smell or taste dysfunction with median durations of $\sim 7$ days (Figure 1.a and Supplementary Figure S.2). The majority (87.3\%) of COVID-19-positive episodes comprised two or more symptoms with varying combinations, which invariably included smell or taste dysfunction (Supplementary Figure S.3). Among COVID-19-negative episodes, the most common symptoms were cough (77.2\%) and shortness of breath (19.6\%), both of which had relatively short median durations of 2 days (IQR 1-5 days) (Figure 1.a, Supplementary Figure S.2). Most (more than two thirds) of COVID-19-negative episodes comprised a single symptom only, the most common of which was cough ( $>50 \%)$ (Figure 1.b).

\section{Discussion}

In this prospective cohort study, we assessed symptomatic and asymptomatic SARS-CoV-2 infections in HCWs in a large tertiary hospital in Mainz, Germany. The seroprevalence of anti-SARS-CoV-2 antibodies ranged from $2.7 \%$ at baseline to $3.8 \%$ at the end of follow-up. Reported seroprevalence of SARS-CoV-2 among HCWs vary across and within settings, which could reflect differences in background community transmission. A recent meta-analysis reported a pooled seroprevalence in HCWs of $8.7 \%$, with the rates ranging from $4 \%$ in Asia to $12.7 \%$ in the US. ${ }^{8}$ In Northern Germany, a prospective study during the early phases of the pandemic reported comparable seroprevalence of anti-SARS-CoV-2 IgG antibodies to our study (4.4\%). ${ }^{9}$ During the same period, lower seroprevalences rates were reported in some regions, ${ }^{10,11}$ whereas a German region with high background rate had an estimated seroprevalence of $15.1 \% .{ }^{12}$ Crosssectional studies that were conducted against a background of high epidemic activity consistently reported higher seroprevalence, such as in Spain $\left(\sim 17 \%\right.$ to $\left.~ 32 \%^{13-15}\right)$, the UK $\left(32 \%^{16}\right)$, and Sweden $\left(19.1 \%{ }^{17}\right)$.

In our university hospital, the seroprevalence among blood donors was estimated to be $0.4 \% .{ }^{18}$ Another study from Germany reported similarly low seroprevalence rates among regular blood donors in three 
different regions. ${ }^{19} \mathrm{~A}$ large cross-sectional study in Central Denmark reported seroprevalence of $3.7 \%$ and $0.6-1.2 \%$ among HCWs and blood donors, respectively. ${ }^{20}$. These results indicate the increased risk of SARS-CoV-2 infection in HCWs.

Our study provided some insights on the duration of anti-SARS-CoV-2 antibody response after natural infection, with apparent waning of antibody titers over time. Out of $98 \mathrm{HCWs}$ who were seropositive at baseline, $\sim 11 \%$ and $\sim 15 \%$ seroreverted by weeks 6 and 12 of follow-up, respectively. In contrast, only one seroreversion occurred among the $33 \mathrm{HCWs}$ who seroconverted by the 2 nd visit. Assuming all seropositive subjects at baseline converted just prior to enrolment, the seroreversion rate in our population would be at most 2.3 per 1000 person-months. A longitudinal study from Italy reported a mean $17 \%$ reduction in IgG titers among seropositive $\mathrm{HCWs}$ within 8 weeks of testing negative for viral RNA. ${ }^{21}$ Another study from Spain reported $68.3 \%$ and $3.1 \%$ of seropositive HCWs seroreverted for IgM and IgG, respectively, after three months of follow-up. ${ }^{22}$

Between August 2020 - January 2021, we observed an increasing rate of SARS-CoV-2 infection, with 33 $(0.9 \%)$ and $35(1.2 \%)$ seroconversions at the $2 \mathrm{nd}$ and $3 \mathrm{rd}$ follow-up visits, respectively. These rates translate to 1.5-2 weekly seroconversions per 1000 persons. Among HCWs with complete follow-up, the overall seroconversion rate in our study would be $2.3 \%$ (68/2925). With the cumulative incidence rate of COVID-19 in our study estimated to be 14.5 per 1000 persons, our findings are consistent with a concurrent regional increase in the background rates of COVID-19. Data reported to the Robert KochInstitute in Germany indicated a ten-fold increase in the cumulative incidence of COVID-19 cases in the Rheinland-Pfalz region (where Mainz is located), from 2.6 per 1000 population in September 2020 to 17.6 per 1000 population in December 2020. ${ }^{23}$

Our study is one of adequately-powered few that were based on prospective design. Our observed seroconversion rate is consistent with findings from the largest prospective study to date, which followed $>12,500 \mathrm{HCWs}$ in the UK over a median of 200 days and reported a seroconversion rate of $0.8 \%{ }^{24}$ Studies from similar settings that reported higher rates were likely underpowered. For example, two relatively small studies from Germany and Denmark reported seroconversion rates of $4.7 \%$ and $4.2 \%$, respectively. ${ }^{25-26}$

Approximately $19 \%$ of HCWs who seroconverted in our study did not report any symptoms. Higher estimates of asymptomatic SARS-CoV-2 infections among HCWs were previously reported. A prospective study in Egypt reported that $64 \%$ of HCWs who seroconverted were asymptomatic. ${ }^{27}$ However, this was based on a single follow-up visit with no active surveillance in-between. In Spain, two cross-sectional studies reported that $49 \%-68 \%$ of seropositive HCWs were asymptomatic. ${ }^{14,15}$ Cross-sectional seroprevalence studies may not be suitable to estimate the true proportion of asymptomatic SARS-CoV-2 infections, given the variable incubation period thereof. Moreover, early clinical presentation of SARS-CoV2 infection is non-specific, and knowledge and awareness thereof are likely to improve over time. Although a low-threshold active surveillance is more sensitive in detecting asymptomatic infections,, this 
approach is resource intensive and may not be suitable in most settings. For example, we had to test $\sim 32$ episodes using RT-PCR to detect one COVID-19-positive episode. Not surprisingly, an even lower proportion (10.2\%) of asymptomatic infections was reported where both symptomatic and asymptomatic HCWs were screened. ${ }^{24}$

The majority (87.3\%) of COVID-19-positive episodes comprised two or more symptoms with varying combinations, which invariably included smell or taste dysfunction. On the other hand, COVID-19 negative episodes were more likely to report single symptoms with relatively short durations, the most common of which was cough. This is consistent with data from other studies. A cross-sectional study on HCWs in Sweden reported up to 28 -fold association between smell or taste dysfunction and seropositive status. ${ }^{17}$

Most COVID-19 cases in our study were nurses with reported direct contact with confirmed COVID-19 cases. In two Scandinavian studies, nurses had the highest risk of infection, and $>3$-fold association was estimated between SARS-CoV-2 infection and direct contact with COVID-19 patients. ${ }^{17,20}$ Similarly, HCWs in Cairo who reported contact with confirmed COVID-19 cases were $>2$-fold at higher risk of infection than those who did not (hazard ratio: 2.2). ${ }^{27}$ None of the COVID-19 cases in our study met either case definition of severe disease, and only one case (1.8\%) required hospitalization. One meta-analysis demonstrated that the risk of severe or critical disease in HCWs was significantly lower than that in nonHCW COVID-19 patients (9.9\% vs. $29.4 \%$, respectively). However, higher prevalence of comorbidities may lead to higher rates of severe disease. In a recent systematic literature review, a higher prevalence of comorbidities in HCWs (18.4\%) could explain an eight-fold higher prevalence of hospitalizations ( 15\%) compared to our study. ${ }^{28}$

A number of limitations need to be mentioned. First, the sampling method was convenience-based, and therefore selection bias could not be ruled out. Reporting of symptoms relied on self-reporting, which may have resulted in recall bias and/or missing data. However, the study team received automated alerts via centralized monitoring system to notify subjects with multiple missing reports. Second, $\sim 18 \%$ of study subjects did not have their scheduled 3rd follow-up visit by the time of the data lock point of this analysis. However, we believe our estimates of seroprevalence and seroconversion at visit 3 are likely conservative, given that more than a third of incident COVID-19 cases occurred shortly before. Last, due to low case positive counts, we could not statistically assess risk factors of SARS-CoV-2 infection, including known sociodemographic and occupational factors which were reported previously. ${ }^{29}$ In addition, the low case count hindered reliable modelling to identify the symptoms most predictive of COVID-19. However, our results suggest that active case finding based on smell or taste dysfunction, with or without other symptoms, would help detect the majority of symptomatic infections.

In conclusion, our findings indicate increasing rates of COVID-19 disease among HCWs in a setting of increased background community transmission. Although we observed an apparent waning in humoral immune response against SARS-CoV-2, we did not detect evidence of reinfections - particularly among those who were seropositive at baseline. We also observed lower than expected rates of asymptomatic 
infections, which highlight the importance of cautious interpretation of reported estimates from crosssectional studies. With the current rolling out of vaccination programs in a setting of increased but regionspecific transmission of SARS-CoV-2 variants of concern, continued monitoring of infection rates is paramount.

\section{Methods}

\section{Study design, setting, and population}

This is a prospective observational cohort study at the University Medical Centre of Mainz, Germany (UM Mainz). The study population comprises a convenience sample of HCWs and medicine/dentistry students, with initial target sample size of 3500. All HCWs at UM Mainz were eligible for enrolment, which took place during August-October 2020. We present interim results from enrolment until 4 January 2021, when subjects were offered enrolment into a phase III COVID-19 vaccine trial.

\section{Study objectives and case definitions}

The primary objectives of the study were to assess the seroprevalence and seroconversion rates of SARSCoV-2 infection, as measured by anti-SARS-CoV-2 antibodies, and the rate of virologically confirmed COVID-19 disease before the introduction of COVID-19 vaccines. A positive serostatus was defined as positive anti-SARS-CoV-2 IgM and/or IgG antibodies, whereas a negative serostatus was defined as testing negative for both antibodies. Seroconversion was defined as seronegative test at baseline and/or any of the follow-up visits followed by seropositive test; and the opposite was applied to define seroreversion. Virologically confirmed COVID-19 disease was defined according to the US Food and Drug Administration (FDA) guidance as an acute illness with positive SARS-CoV-2 RT-PCR test and at least one symptom suggestive of COVID-19 (Supplementary Table S.1). Secondary outcomes included the rates of COVID-19 disease based on a more specific case definition by the Coalition for Epidemic Preparedness Innovations (CEPI), suspected COVID-19 disease, and severe COVID-19 disease (Supplementary Table S.1). Symptoms occurring between four days before the index RT-PCR test and four days after the last confirmatory RT-PCR test were defined as a single episode.

\section{Procedures}

At baseline, study subjects were scheduled for five follow up visits, in which data on demographics, occupational information, and risk factors of SARS-CoV-2 infection were collected (Supplementary Tables S.2-S.4). Serum samples were collected at baseline and in all follow-up visits for serological two-step testing of anti-SARS-CoV-2 IgG and IgM antibodies: first using ARCHITECT ${ }^{\circledR}$ i2000SR (Abbott Laboratories), and if positive or borderline-positive, re-tested using Elecsys ${ }^{\circledR}$ Anti-SARS-CoV-2 (Roche Diagnostics). 
In addition, daily self-reported data on symptoms suggestive of COVID-19 disease were collected via a mobile phone application (TrialPa/® Integra IT; Supplementary Table S.5). All study subjects received automated daily push-notifications as reminders, and those with multiple missing reports were contacted by the study site to ensure adherence. Subjects who reported cough, shortness of breath, chills, smell or taste dysfunction, and/or fever (defined as a body temperature $\geq 37.8^{\circ} \mathrm{C}$ ) were then screened by trained study personnel using scripted phone interviews to establish eligibility for virological confirmation.

Eligible study subjects were then invited to the study site for SARS-CoV-2 RT-PCR testing using selfcollected nasopharyngeal swab samples. Next, an algorithm was applied based on the index test result (Supplementary Figure S.1). Briefly, subjects who tested positive for SARS-CoV-2 were interviewed for the collection of data including clinical course and outcome (Supplementary Table S.6). Follow-up tests were scheduled after 21 days, then weekly until resolution, which was defined as two consecutive negative RTPCR tests. Similarly, those who initially tested negative for RT-PCR were invited for a confirmatory test 24 hours after the index test.

\section{Statistical analysis}

The seroprevalence of anti-SARS-CoV-2 antibodies and the corresponding 95\% confidence intervals (Cls) were estimated using the Clopper-Pearson interval exact method, and the Rogan-Gladen method was used to adjust the crude seroprevalence for diagnostic misclassification. Seroconversion was calculated as the percentage of subjects who were seronegative at baseline and/or any of the follow-up visits and later became seropositive. The incidence rates (per 1000 person-months) of virologically confirmed COVID-19, including severe disease, were estimated using the Garwood's method. The person-time at-risk was calculated from baseline to end of study for non-COVID-19 subjects and from baseline to the date of virological confirmation for COVID-19 cases. All statistical analyses were carried out using R statistical software.

\section{Ethical approvals}

The study was approved by the Independent Ethics Committee at UM Mainz and all methods were performed in accordance with the Declaration of Helsinki. All study subjects signed an informed consent form (ICF) (in German) prior to participation. All signed and dated ICFs were available for verification by study monitors (Harmony Clinical Research). The study is registered in the European Union's electronic register of Post-Authorization Studies (EU PAS register number EUPAS37174) and is expected to continue until early 2022.

\section{Declarations}

\section{Acknowledgements}


This study is supported by CureVac AG, Tübingen, Germany.

The authors would like to thank the staff in UM Mainz and IZKS for their efforts. We thank Els Michels and Tom van Paepegem at Harmony Clinical Research who carried out the study monitoring. We also thank Tom De Smedt (P95) for GDPR compliance management, Thao Tran (P95) for statistical assistance, and the team at Integra IT for their support.

\section{Author contributions}

TV, OO, WHM, and LO developed the study protocol. SG and FK in their role as Principal Investigator and Co-Investigator collected the data with support from TE, DS, CJ, and AMK. AT performed the statistical analyses. $\mathrm{OO}$ wrote the manuscript. $\mathrm{LO}$ and all authors reviewed the manuscript and approved it.

\section{Competing interests}

TV is the managing director of P95, and also a paid consultant at CureVac AG, fulfilling the role of safety physician. SG and FK are employees of UM Mainz and in addition paid for this study as principal investigator and co-investigator, respectively, by CureVac AG. DS, CJ, and AMK are employees of UM Mainz. LO is an employee of CureVac AG. OO, AT and WHM are employees of P95; all state no conflict of interest.

\section{Data availability}

The datasets generated during and/or analyzed during the current study are available from the corresponding author on reasonable request.

\section{References}

1. Zhu, N. et al. A Novel Coronavirus from Patients with Pneumonia in China, 2019. N Engl J Med, 382, 727-733 https://doi.org/10.1056/NEJMoa2001017 (2020).

2. The World Health Organization. Coronavirus disease 2019 (COVID-19) Situation Reports 11, 22, and 52. (WHO, 2020).

3. The World Health Organization. WHO Coronavirus Disease (COVID-19) Dashboard, (2021).

4. Iversen, K. et al. Risk of COVID-19 in health-care workers in Denmark: an observational cohort study. Lancet Infect Dis, 20, 1401-1408 https://doi.org/10.1016/s1473-3099(20)30589-2 (2020).

5. Gomez-Ochoa, S. A. et al. COVID-19 in Health-Care Workers: A Living Systematic Review and MetaAnalysis of Prevalence, Risk Factors, Clinical Characteristics, and Outcomes. Am J Epidemiol, 190, 161-175 https://doi.org/10.1093/aje/kwaa191 (2021). 
6. Dooling, K. et al. The Advisory Committee on Immunization Practices' Interim Recommendation for Allocating Initial Supplies of COVID-19 Vaccine - United States, 2020. MMWR Morb Mortal Wkly Rep, 69, 1857-1859 https://doi.org/10.15585/mmwr.mm6949e1 (2020).

7. Verger, P. et al. Attitudes of healthcare workers towards COVID-19 vaccination: a survey in France and French-speaking parts of Belgium and Canada, 2020. Euro Surveill, 26, https://doi.org/10.2807/15607917.ES.2021.26.3.2002047 (2021).

8. Galanis, P., Vraka, I., Fragkou, D., Bilali, A. \& Kaitelidou, D. Seroprevalence of SARS-CoV-2 antibodies and associated factors in healthcare workers: a systematic review and meta-analysis. $J$ Hosp Infect, 108, 120-134 https://doi.org/10.1016/j.jhin.2020.11.008 (2021).

9. Herzberg, J. et al. Prospective Sero-epidemiological Evaluation of SARS-CoV-2 among Health Care Workers in a German Secondary Care Hospital. Int J Infect Dis, 102, 136-143 https://doi.org/10.1016/j.ijid.2020.10.026 (2021).

10. Brehm, T. T. et al. Seroprevalence of SARS-CoV-2 antibodies among hospital workers in a German tertiary care center: A sequential follow-up study. Int J Hyg Environ Health, 232, 113671 https://doi.org/10.1016/j.jheh.2020.113671 (2021).

11. Korth, J. et al. SARS-CoV-2-specific antibody detection in healthcare workers in Germany with direct contact to COVID-19 patients. J Clin Virol, 128, 104437 https://doi.org/10.1016/j.jcv.2020.104437 (2020).

12. Finkenzeller, T. et al. [SARS-CoV-2 antibodies in ICU and clinic staff: From Germany's region with the highest infection rate]. Med Klin Intensivmed Notfmed, 115, 139-145 https://doi.org/10.1007/s00063-020-00761-5 (2020).

13. Escribano Ceruelo, E. et al. Seroprevalence of antibodies against SARS-CoV-2 among health care workers in a pediatric monographic hospital in Madrid (Spain). Enferm Infecc Microbiol Clin, https://doi.org/10.1016/j.eimc.2020.12.011 (2021).

14. Garralda Fernandez, J. et al. Impact of SARS-CoV-2 pandemic among health care workers in a secondary teaching hospital in Spain. PLoS One, 16, e0245001 https://doi.org/10.1371/journal.pone.0245001 (2021).

15. Galán, M. I. et al. Hospital-Wide SARS-CoV-2 seroprevalence in health care workers in a Spanish teaching hospital. Enferm Infecc Microbiol Clin, https://doi.org/10.1016/j.eimc.2020.11.015 (2020).

16. Grant, J. J. et al. Seroprevalence of SARS-CoV-2 antibodies in healthcare workers at a London NHS Trust. Infect Control Hosp Epidemiol, 42, 212-214 https://doi.org/10.1017/ice.2020.402 (2021).

17. Rudberg, A. S. et al. SARS-CoV-2 exposure, symptoms and seroprevalence in healthcare workers in Sweden. Nat Commun, 11, 5064 https://doi.org/10.1038/s41467-020-18848-0 (2020).

18. Runkel, S. et al. Prevalence of Severe Acute Respiratory Syndrome Coronavirus-2-specific Antibodies in German Blood Donors during the COVID-19 Pandemic. Clin Lab, 66, https://doi.org/10.7754/Clin.Lab.2020.200915 (2020).

19. Fischer, B., Knabbe, C. \& Vollmer, T. SARS-CoV-2 IgG seroprevalence in blood donors located in three different federal states, Germany, March to June 2020. Euro Surveill, 25, 
https://doi.org/10.2807/1560-7917.ES.2020.25.28.2001285 (2020).

20. Jespersen, S. et al. SARS-CoV-2 seroprevalence survey among 17,971 healthcare and administrative personnel at hospitals, pre-hospital services, and specialist practitioners in the Central Denmark Region. Clin Infect Dis, https://doi.org/10.1093/cid/ciaa1471 (2020).

21. De Carlo, A. et al. SARS-COV-2 Serological Profile in Healthcare Professionals of a Southern Italy Hospital. Int J Environ Res Public Health, 17, https://doi.org/10.3390/ijerph17249324 (2020).

22. Moncunill, G. et al. SARS-CoV-2 Seroprevalence and Antibody Kinetics Among Health Care Workers in a Spanish Hospital After 3 Months of Follow-up. J Infect Dis, 223, 62-71 https://doi.org/10.1093/infdis/jiaa696 (2021).

23. Koch-Institute, R. Coronavirus Disease 2019 (COVID-19) - Daily Situation Reports(2020).

24. Lumley, S. F. et al. Antibody Status and Incidence of SARS-CoV-2 Infection in Health Care Workers. $N$ Engl J Med, 384, 533-540 https://doi.org/10.1056/NEJMoa2034545 (2021).

25. Weinberger, T. et al. Prospective Longitudinal Serosurvey of Health Care Workers in the First Wave of the SARS-CoV-2 Pandemic in a Quaternary Care Hospital in Munich, Germany. Clin Infect Dis, https://doi.org/10.1093/cid/ciaa1935 (2021).

26. Rasmussen, K. M. B. et al. COVID-19 infection rate among tertiary referral center otorhinolaryngology healthcare workers. Eur Arch Otorhinolaryngol, 1-8 https://doi.org/10.1007/s00405-021-06615-w (2021).

27. Mostafa, A. et al. SARS-CoV-2 seroconversion among 4040 Egyptian healthcare workers in 12 resource-limited healthcare facilities: A prospective cohort study. Int J Infect Dis, 104, 534-542 https://doi.org/10.1016/j.jij.2021.01.037 (2021).

28. Gholami, M. et al. COVID-19 and healthcare workers: A systematic review and meta-analysis. Int J Infect Dis, 104, 335-346 https://doi.org/10.1016/j.ijid.2021.01.013 (2021).

29. Lan, F. Y. et al. Sociodemographic risk factors for COVID-19 infection among Massachusetts healthcare workers: a retrospective cohort study. Infect Control Hosp Epidemiol, 1-23 https://doi.org/10.1017/ice.2021.17 (2021).

\section{Figures}



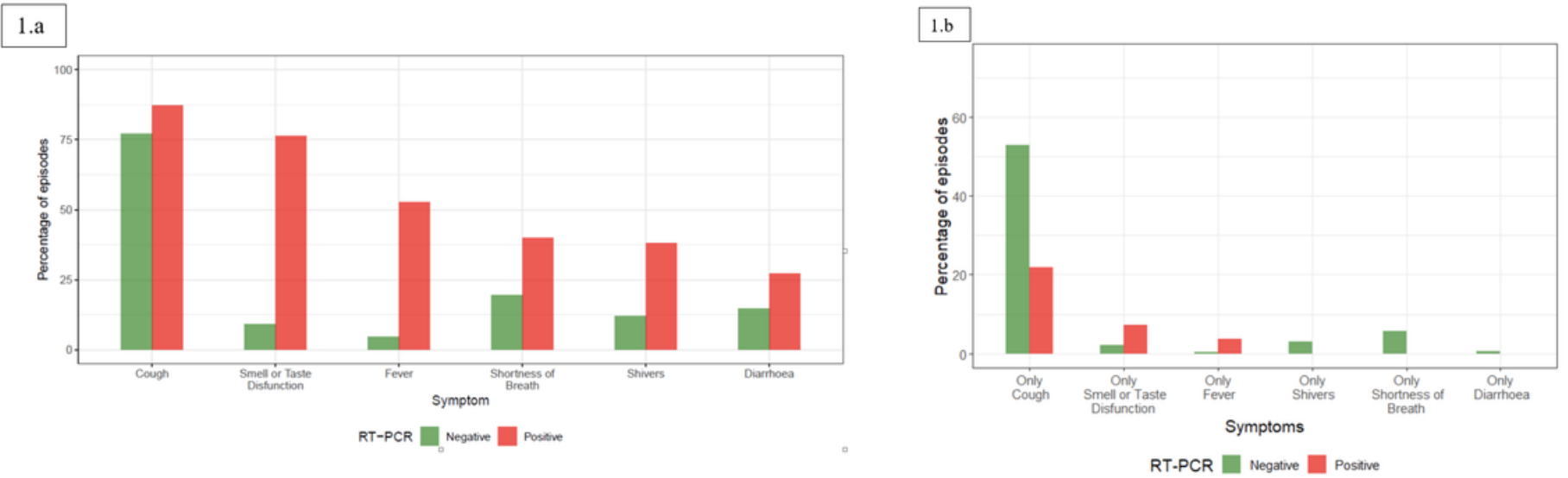

\section{Figure 1}

Symptoms reported by clinically suspected COVID-19 cases among HCWs in the CPMprevac study by RTPCR test status. Figure 1.a. With or without other symptoms per episode (green bars: RT-PCR negative, red bars: RT-positive) Figure 1.b. Without other symptoms per episode (i.e., single-symptom episodes) (green bars: RT-PCR negative, red bars: RT-positive)

\section{Supplementary Files}

This is a list of supplementary files associated with this preprint. Click to download.

- CPMprevacsupplementsFinalSR.pdf 